\title{
Alterações orais em crianças prematuras e de baixo peso ao nascer: a importância do trabalho multidisciplinar
}

Oral alterations in preterm and low birth weight children: the importance of multidisciplinary work

Alteraciones bucales en niños prematuros y de bajo peso al nacer: la importancia del trabajo multidisciplinar

Recebido: 03/02/2022 | Revisado: 09/02/2022 | Aceito: 13/02/2022 | Publicado: 20/02/2022

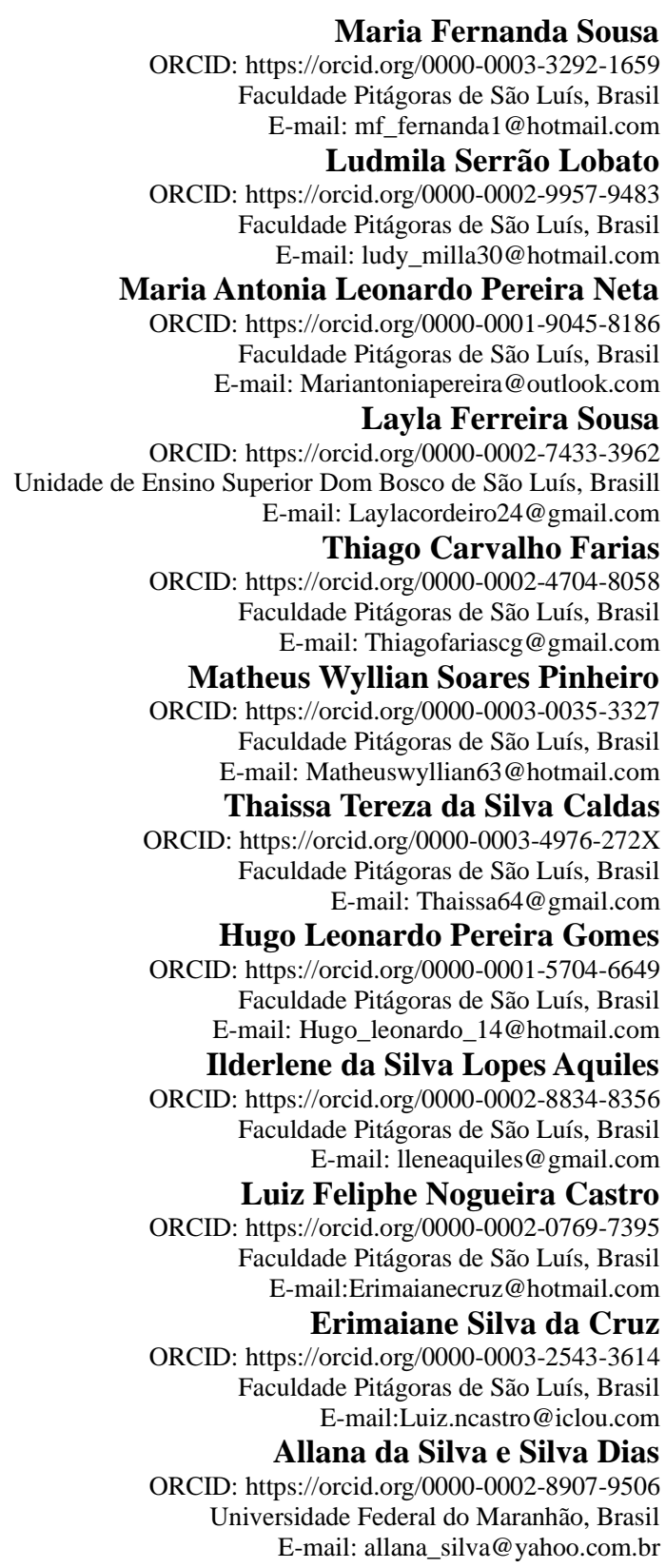

\section{Resumo}

Objetivo: realizar uma revisão de literatura atualizada e disponível corelacionando alterações bucais e prematuridade com baixo peso ao nascer e apresentar a importância da atuação multidisciplinar. Revisão de literatura: A literatura tem exposto correlação entre alterações orais e parto prematuro, crianças que apresentam essa condição podem 
manifestar: defeitos de esmalte, aumento na incidência da doença cárie, atraso na cronologia de erupção decídua, além de alterações no palato e arcada dentária. Essas alterações prejudica o desenvolvimento da criança seja no quesito estético, emocional e funcional. Considerações finais: Em síntese, torna-se importante uma abordagem interdisciplinar entre pediatras e odontopediatras, pois as manifestações bucais podem ser geradas e agravadas por condições sistêmicas. Cabe à equipe multidisciplinar educar, prevenir e reduzir possíveis alterações bucais em crianças prematuras.

Palavras-chave: Recém-nascido; Baixo peso; Cárie dentária; Manifestações bucais; Dente decíduo.

\begin{abstract}
Objective: carry out a review of the available and updated literature that relates oral alterations and prematurity with low birth weight and present the importance of multidisciplinary action. Literature review: Literature has shown a correlation between oral alterations and preterm birth, children with this condition may manifest: enamel defects, increased incidence of caries disease, delay in the chronology of deciduous eruption, in addition to alterations in the palate and dental arch. These changes affect the child's development in terms of aesthetic, emotional and functional aspects. Final considerations: In summary, an interdisciplinary approach between pediatricians and pediatric dentists is important, as oral manifestations can be generated and aggravated by systemic conditions. It is up to the multidisciplinary team to educate, prevent and reduce possible oral changes in premature children.
\end{abstract}

Keywords: Newborn; Low birth weight; Dental caries; Oral manifestations; Primary tooth.

\title{
Resumen
}

Objetivo: realizar una revisión de la literatura disponible y actualizada que relaciona las alteraciones orales y la prematuridad con el bajo peso al nacer y presentar la importancia de la acción multidisciplinar Revision de literatura: La literatura ha expuesto una correlación entre las alteraciones orales y el parto prematuro, los niños que presentan esta condición pueden manifestar: defectos del esmalte, aumento de la incidencia de caries, retraso en la cronología de la erupción decidua, además de alteraciones en el paladar y arco dentario. Estos cambios perjudican el desarrollo del niño en términos de aspectos estéticos, emocionales y funcionales.Consideraciones finales: En resumen, es importante un abordaje interdisciplinario entre pediatras y odontopediatras, ya que las manifestaciones bucales pueden ser generadas y agravadas por condiciones sistémicas. Corresponde al equipo multidisciplinario educar, prevenir y reducir los posibles cambios bucales en los niños prematuros.

Palabras clave: Recién nacido; Bajo peso al nacer; Caries; Manifestaciones bucales; Dentición temporal.

\section{Introdução}

O nascimento prematuro é classificado por aquele que antecede às 37 semanas de gestação. Já o baixo peso ao nascer pode ser determinado por $(<2500 \mathrm{~g})$ ao nascimento. A origem da prematuridade e do baixo peso ao nascer é considerada múltipla e envolve diversos fatores podendo ser de cunho genético, obstétrico, nutricional, ambiental, econômico e outros (Williams et al.,2000).

O monitoramento recorrente da prematuridade é de suma importância devido a mortalidade e morbidade, a partir da Lei $N^{\circ} 6.015$ de 31 de dezembro de 1973 o Brasil passou a ter uma fonte de estatísticas sobre nascidos vivos, com principal objetivo analisar as estatísticas e assim possibilitar a aplicação de condutas básicas de saúde. Nos últimos três anos observouse um aumento no percentual segundo Dados do sistema de nascidos vivos (SINASC) em 2020 registrou-se $8,6 \%$ o que em 2005 variava em torno de 6,6(Silveira et al., 2009).

Esses dados se tornam inquietantes por conta dos problemas que essas crianças possam apresentar, como o desenvolvimento incorreto do sistema estomatognático que é responsável pelo desenvolvimento dos dentes, lábios, língua, bochechas, glândulas, artérias, veias e nervos, que realizam funções de sucção, mastigação, deglutição, fonoarticulação e respiração, e dessa forma, acabar desencadeando alterações orais. Entre as alterações mais comuns que essas crianças podem manifestar estão: defeitos de esmalte, aumento na incidência da doença cárie, atraso na cronologia de erupção decídua, além de alterações no palato e arcada dentária. (Cortines et al.; 2016).

Devido à complexidade no atendimento e aos fatores de risco que essas crianças estão sujeitas, é de suma necessidade que se forneça atenção integral com toda a equipe e saúde bucal para que tenham conhecimento sobre as possíveis alterações orais e dessa maneira possibilitar melhor tratamento com finalidade de promover melhor qualidade de vida para essas crianças (Viana et al.,2013; Cruvinel et al.,2010). 
Dessa forma, baseado que foi expresso anteriormente o presente trabalho tem como finalidade, por meio de uma revisão narrativa de literatura evidenciar as principais alterações bucais que acometem as crianças nascidas a pré-termo e/ou com baixo peso ao nascer e expor a importância da atuação efetiva da equipe multidisciplinar no amparo ao prematuro.

\section{Metodologia}

O presente trabalho refere-se a uma revisão narrativa de literatura, em que as buscas bibliográficas se fundaram através de seleções de artigos publicados nos idiomas: português, inglês e espanhol, que se encontravam disponibilizados nas bases de dados eletrônicos PubMed,Lilacs, Google Acadêmico e Scielo. Além disso, os artigos incluídos foram sem data limite de publicação. Os descritores utilizados em combinação com os operadores booleanos para a pesquisa foram: "Recémnascido", "baixo peso", "cárie dentária”, "manifestações bucais" e "dente decíduo" indexados no Decs e "newborn” , "Low weight", "dental cavity", "oral manifestations"”, deciduous tooth" indexados no Mash.

Figura 1. Fluxograma com a quantidade de artigos obtidos a partir da filtragem nas bases de dados.

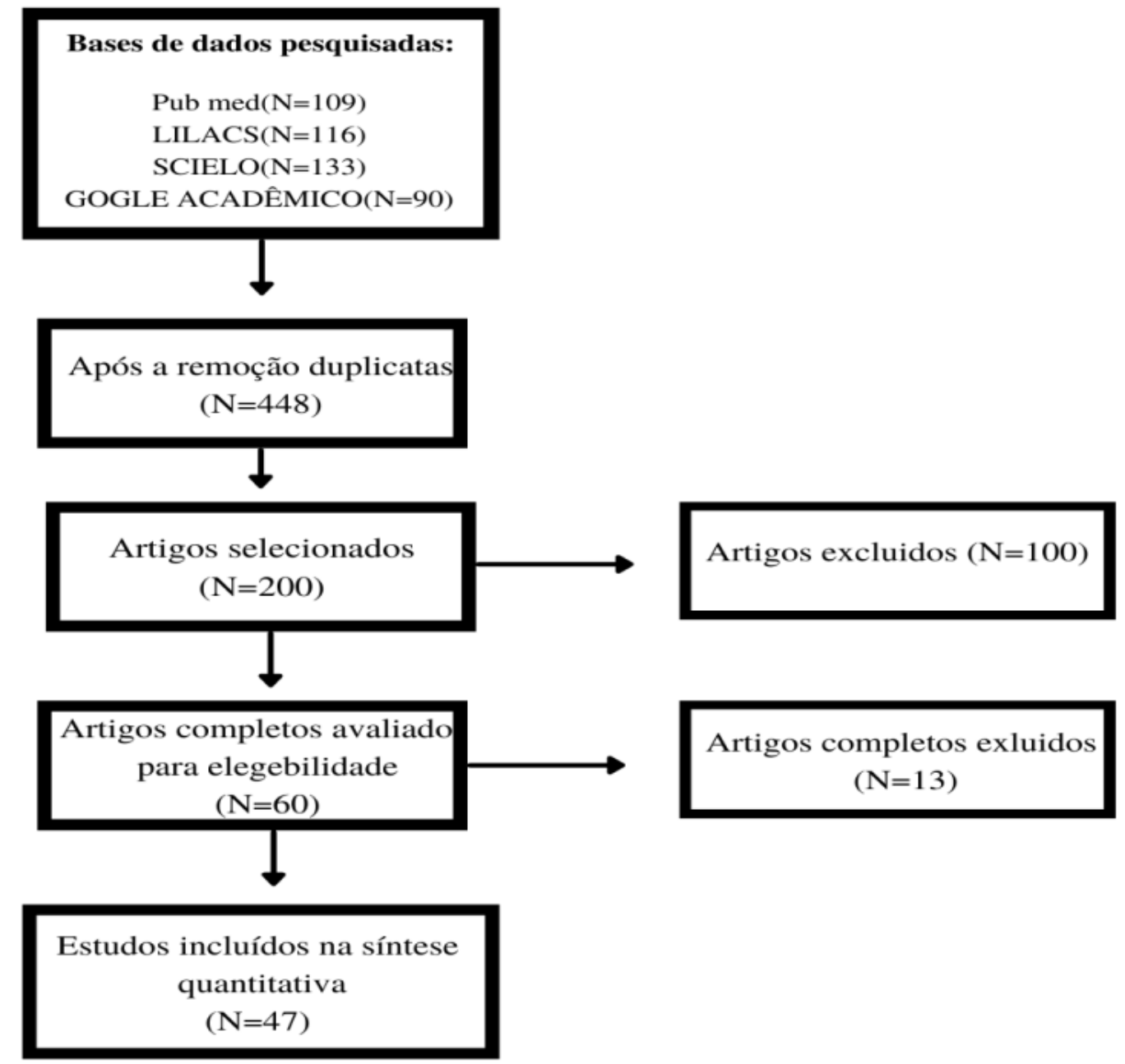

Fonte: Autores.

Em conformidade com, Rother (2007) A revisão de literatura narrativa consiste em produções ampliadas e elaboradas, apontadas como cabíveis para a definição e discussão do desenvolvimento de uma estabelecida temática, de um panorama teorético e contextual. Atentando que o ledor obtenha compreensão e se renove em torno do assunto em uma breve 
duração.

A eleição de estudos selecionados para elaboração da presente revisão de literatura se deu por meio de títulos e resumos. Os critérios de inclusão que conduziram sua produção foram através da busca por trabalhos com relevância e associação com a pesquisa que foi realizada, que se relaciona à temática de alterações orais em crianças prematuras e de baixo peso ao nascer: a importância do trabalho multidisciplinar. A pesquisa abrangeu estudos clínicos, relatos de casos e literatura cinza (monografias, teses e dissertações). A tabela 1 apresenta resumidamente os artigos obtidos depois da filtragem e que contribuíram para a elaboração do "corpus" da pesquisa e foram utilizados de fato para a realização das discussões e para se chegar a conclusão.

Tabela 1. Artigos obtidos após as filtragens e, que contribuíram o "corpus" da pesquisa.

\begin{tabular}{|c|c|c|}
\hline Autores & $\begin{array}{l}\text { Ano de } \\
\text { publicação }\end{array}$ & Titulo \\
\hline Seow WK, Brown J, Tudehope D, Callaghan M. & 1994 & $\begin{array}{l}\text { Development defects in the primary dentition of lowbirth weight: adverse } \\
\text { effects of laryngoscopy and prolonged endotracheal intubation. }\end{array}$ \\
\hline Fadavi S, Punwani I, Vidyasagar D & 1993 & Prevalence of dental caries in prematurelyborn children \\
\hline Caixeta fb, pires Corrêa. & 2005 & $\begin{array}{l}\text { OS DEFEITOS DO ESMALTE E A ERUPÇÃO DENTÁRIA EM } \\
\text { CRIANÇAS PREMATURAS. }\end{array}$ \\
\hline Saraiva MC, Bettiol H, Barbieri MA, Silva AA & 2007 & intrauterine growth restriction and preterm birth associated with dental caries? \\
\hline Nicolau B, Marcenes W, Bartley M, Sheiham A. & 2003 & $\begin{array}{l}\text { A life course approach to assessing causes of dental caries experience: the } \\
\text { relationship between biological, behavioural, socio-economic and } \\
\text { psychological conditions and caries in adolescents. }\end{array}$ \\
\hline Rajshekar AS, Laxminarayan N. & 2011 & $\begin{array}{l}\text { Comparison of primary dentition caries experience in pre-term low birth- } \\
\text { weight and full-term normal birth-weight children aged one to six years. }\end{array}$ \\
\hline Harila-Kaera V, Grön M, Heikkinen T, Alvesalo L. & 2002 & Sagittal occlusal relationships and asymmetry in prematurely born children. \\
\hline
\end{tabular}

Fonte: Autores.

\section{Resultados}

\section{Alterações orais}

\section{Defeitos no esmalte dentário}

O nascimento pré-termo tem sido referido como uma das origens para o atraso na cronologia de erupção dentária e surgimento de defeitos no esmalte (Brasil - Ministério da Saúde. DATAS 1994). Uma das circunstâncias que podem justificar o surgimento de defeitos no esmalte é a imaturidade de alguns órgãos, como rins, glândulas paratireoide e fígado no processo de suplementação de cálcio e fósforo. Dessa forma sua metabolização fica prejudicada e ocasiona o aparecimento de lesões (Eid, 2020; Grahnen et al.,1974; Koch et al.,1987).

A hipoplasia é vista como a formação insuficiente de matriz orgânica do dente (Aine L et al.,2000). ”clinicamente manifesta-se com falta total ou parcial da superfície de esmalte, apresentando uma estética insatisfatória, dentes sensíveis, má-oclusão, bem como predisposição à cárie dental ."( Seow, 1991).

Enquanto a hipocalcificação ou opacidade do esmalte sucede de meios que decorrem da calcificação dentária e justificar o surgimento de defeitos no esmalte é a imaturidade de alguns órgãos, como rins, glândulas paratireoide e fígado no processo de suplementação de cálcio e fósforo. Dessa forma sua metabolização fica prejudicada e ocasiona o aparecimento de lesões (Eid ,2020; Grahnen et al.,1974; Koch et al.,1987).Clinicamente podem resultar em lesões com áreas branco-opacas, amareladas ou acastanhas (Aine et al.,2000). 
(Caixeta et al., 2005) Observou em um estudo com 100 crianças prematuras de 06 messes á 06 anos que 35\% delas apresentavam defeitos no esmalte e $65 \%$ não apresentava. Foi visto também neste trabalho que defeitos de esmalte apareceram em $66,7 \%$ das crianças entubadas por menos de duas horas comparadas com 74,2\% entubadas entre 2 e 64 horas.

Segundo (Pinkham,1996), descreveu que crianças nascidas a pré-termo tem maior predisposição ao desenvolvimento de alterações no esmalte dentário devido a danos traumáticos associados à laringoscopia e intubação orotraqueal, visto que a amelogênese está em uma fase analítico. (Norén et al.,1993) fez um estudo em que selecionaram 35 pacientes que estiveram entubados até os 3(três) messes de idade. Os quais foram acompanhados e examinados até os 3 (três) anos de idade. Foi observado que 26 desses pacientes apresentaram hipoplasia e opacidade do esmalte tendo maior ocorrência do lado direito do arco superior. Sugeriu ainda que havia existência de um agente traumático, causando pressão sobre o rebordo alveolar anterior e predispondo à ocorrência de hipoplasia.

\section{Cárie dentária}

A reação entre cárie dentária e prematuridade com baixo peso ao nascer pode ser expressa pela debilitada resposta imunológica dessas crianças até os 5 (cinco) anos de idade e torna esse grupo mais predisposto a infecção por Streptococcus mutans (Chandra et al.,2005;Wan et al.,2003). Dentre as condições de risco que levam essas crianças ao desenvolvimento da doença cárie ,estão: aleitamento artificial, exposição a níveis elevados de sacarose, condições nutricionais, práticas incorretas de higiene oral, entre outros (Fadavi et al.,1993;Saraiva et al.,2007).

A enfraquecida condição nutricional, constantemente vista nessas crianças, pode elevar a disposição ao desenvolvimento da cárie ao atingir as glândulas salivares com a redução do fluxo salivar, alteração da composição da saliva e, como consequência afetar a capacidade tampão (Psoter et al., 2005).Crianças nascidas a pré-termo manifestam ainda motricidade oral comprometida que acaba dificultando a amamentação, desse modo, o leite materno é substituído pelo aleitamento artificial oferecido por meio de mamadeiras (Bertoncelli et al.,2012). O aleitamento artificial possui açúcar em sua fórmula, o habito duradouro especialmente no período noturno eleva o risco de cárie dentária (Diniz et al.,2011).O açúcar extrínseco adicionado é substrato para as bactérias bucais correlacionados a condições etiológicas (Viana et al., 2013).

\section{Atraso na cronologia de erupção}

Acredita-se que o nascimento prematuro e de baixo peso ao nascer seja um dos fatores que possam levar ao atraso na cronologia de erupção dentária (Seow et al.,1988;Viscardi et al., 1994) . Constatou-se que crianças nascida a pré-termo vem ocorrer à erupção dos primeiros dentes por volta da $39^{\mathrm{a}}$ semana, em contrapartida as nascidas a termo na $30^{\mathrm{a}}$ semana (Seow et al.,1991;Seow et al.,1984). Bebes com menor idade gestacional e menor peso ao nascer está propenso a terem a erupção do $1^{\circ}$ dente de forma tardia, uma vez que o desenvolvimento físico está intimamente ligado com a cronologia de erupção (Fadavi et al.,1992).

O baixo peso ao nascer pode ser um fator que possa intensificar esse atraso, em geral, devido a um déficit nutricional. Uma vez havendo deficiência do cálcio que pode incluir em alterações estruturais e morfológicas do elemento dentário ( Ferrini et al., 2007 ; Castro et al.,2019). Condições nutricionais influem consideravelmente na odontogênese dentária e erupção dos dentes, pois as vitaminas são intermediadores metabólicos essenciais (Seow et al., 2000)

Alguns mecanismos na vida pós-natais acabam intervindo para melhor no processo de erupção dentaria. Podendo ser a amamentação, pois supre qualquer deficiência nutritiva que essa criança possa enfrentar. Além de o emprego consciente na utilização de mordedores e mamadeiras, pois ajudam na esfoliação dentária e também intervém nos mecanismos funcionais distintos da musculatura orofacial (Neto et al.,2014; Dadalto et al.,2018).É importante ressaltar que crianças com idade gestacional corrigida não se nota atraso na cronologia de erupção (Seow ,1991; Paulsson et al.,2004). 


\section{Alterações no palato e arcada}

Constantemente nota-se a existência de deficiência respiratória em bebês que nascem prematuros e acaba sendo necessária a utilização de ventilação mecânica por meio de tubos nasotraqueais ou orotraaqueais. Através dessa intervenção verifica-se anomalias á laringe que acaba ocasionando o surgimento de cistos subglóticos, estenose bronquial, fendas palatinas, ranhuras alveolares, dilaceração dos dentes decíduos, mordida cruzada, mordida aberta, problemas na fala e defeitos no esmalte (Seow et al., 1984).

Observa-se aumento na prevalência ao desenvolvimento de fendas palatais em crianças prematuras e com baixo peso ao nascer (Diniz et al.,2011 ; Cortines et al., 2016). (Duke et al., 1976) verificou a presença de alterações orais em recém nascidos após 70 horas de intubação. (Erenberg et al.,1984). Constataram que já nota surgimento de danos palatais após 12 horas de intubação. Em consequência da pressão criada pela cânula orotraqueal, nota-se que o desenvolvimento do palato fica acometido e lesa o processo lateral. Dessa forma, verifica-se a construção de um palato com algumas características anormais, como por exemplo: menor, mais fundo e mais estreitado (Diniz et al.,2011; Cortines et al., 2016). Estudos tem recomendado o uso de posicionadores intraorais com objetivo de equilibrar a cânula orotraqueal, amenizando assim a pressão e gerada pela mesma, dessa forma evitando que ocorra traumas e deformidades orais (Erenberg et al.,1984).

Indicadores revelaram que o grupo de crianças do sexo masculino exibe maior predominância ao desenvolvimento de fendas palatinas e/ou fissuras labiais quando comparadas ao grupo do sexo feminino, sendo 1,3 vezes maior (Shibukawa et al.,2019).

As mutações anatômicas no palato são capazes de causar um enorme resultado de más oclusões, sendo elas mordidas cruzadas e anomalias no trajeto da erupção dentária e por meio disso gerar o desalinhamento dos dentes e dessa maneira gerar o carecimento maior ao tratamento ortodôntico (Cortines et al.,2016).

\section{Como deve atuar a equipe multidisciplinar?}

Devido à complexidade no atendimento das crianças prematuras e/ou baixo peso ao nascer, torna-se de extrema importância atuação da equipe multidisciplinar. A interação de todos os profissionais é de extremo valor e deve acontecer de forma organizada. Os cuidados neonatais têm obtido maior êxito nos últimos anos o que tem favorecido as condições de supervivência das crianças com menor idade gestacional e/ou peso ao nascer (Machado et al., 2004).O atendimento da equipe de saúde precisa começar no pós-natal, e faz-se importante a inclusão do pré-natal odontológico como medida preventiva ás possíveis alterações que se possam desencadear.

Entretanto a saúde bucal dessas crianças tem sido negligenciada por parte da família e equipe de saúde. O que tem favorecido o aparecimento de anomalias orais principalmente aumento na predisposição a lesões de cárie. (Pierce et al.,2002) Observou que exames orais pode ser naturalmente introduzido na rotina pediátrica.Tendo em mente que a educação em saúde oral precisa acontecer em seguida do nascimento, é de suma importância que haja interação entre pediatras e odontopediatras, para que seja traçado um plano de tratamento com adaptação entre saúde bucal e geral. Visto que o primeiro convívio que essas crianças tem com a equipe de saúde é por parte do pediatra (Sheiham et al., 1992).

Dessa forma, propõe-se que os pediatras estão capacitados para instruir os pais a cerca dos cuidados preventivos referentes à doença cárie, higiene oral e uso correto de flúor para cada idade. As crianças prematuras tendem a serem mais ansiosas e agitadas, assim devem ser encaminhas para o trabalho odontológico especializado para que a sua experiência seja mais calma e menos traumática (Brogårdh-Roth et al.,2008). 


\section{Discussão}

(Pinkham,1996) Assegurou que crianças nascidas a pré-termo tem maior predisposição ao desenvolvimento de distúrbios buco-dentários quando comparados as nascidas a termo, por causa de um maior número de alterações que estão submetidos no período neonatal. Ainda que ocorra inúmeras disposições sistêmicas durante o período neonatal, como por exemplo: infecções, problemas respiratórios, raquitismo e outros. Em relação a crianças prematuras e a hipoplasia do esmalte é imprescindível excluir que haja uma correlação e é notória a relevância de cada condição, uma vez que se observa que ocorrem de forma conjunta. Além de tudo, é possível que esses distúrbios ajam por meio de uma pratica em conjunto, diminuindo o suprimento mineral e atingindo diretamente a mineralização dos tecidos duros dentários. (SEOW et al., 1984; SEOW et al., 1989).

(Fadavi et al. 1993; Lai et al. 1997) observaram que havia correlação entre hipoplasia do esmalte e maior prevalência da doença cárie em crianças prematuras, condições como a utilização prolongada de mamadeira, o baixo nível socioeconômico, além da situação nutricional e a inexistência de ações preventivas, como a prática correta de higiene oral e visitas periódicas ao dentista é de suma importância a serem resaltados, uma vez que a caria dentária é de caráter multifatorial.

É importante resaltar que a literatura mostra desiguais índices $56 \%$ a $12,5 \%$ de predomínio da doença carie em prematuros (Júnior et al.,2014;Rajshekar et al.,2011).Os estudos ainda tem se mostrado controversa quando a associação entre cárie dentaria e prematuridade.( Nicolau et al.,2003)referiram ligação entre prematuridade e/ou baixo peso as nascer com a doença cárie. Porém, (Saraiva et $a$ l.,2007) não achou evidencias que comprovasse essa junção.

(Harila-Kaera et al.,2002) Consideraram que crianças nascidas a pré-termo podem exibir maior potencial a agentes etiológicos que propiciam o surgimento de mordida aberta anterior, como hábitos de sucção e nutrição, infecções respiratórias, respiração oral e por via nasal inadequada além de práticas orais não nutritivos.

(Caixeta \& Corrêa, 2005) Recomendaram a realização de exames pós-natal constante e a realização de instruções por parte do médico pediatra sobre a absorção de calcio nessas crianças nascidas prematuramente. Impossibilitando que assim não haja implicações na fase de decomposição e mineralização do esmalte.

A educação em saúde bucal e o emprego de medidas preventivas devem ser incorporada para essa classe de crianças nascidas prematuramente, visto que as mutações na superfície do esmalte, mesmo estando imperceptível clinicamente, exibem microporosidades, a qual pode gerar crescimento na susceptibilidade ao desenvolvimento de lesões de cárie(NORÉN, 1983).

\section{Conclusão}

Com os avanços tecnológicos nas áreas de obstetrícia e nos cuidados pós e pré-natais vem se observando menor índice de mortalidade em crianças nascidas prematuras e/ou com baixo peso ao nascer. Portanto, faz-se necessário a criação de programas que atendam a crianças integralmente desde a vida intrauterina até o pós-natal. Deve ser traçado um planejamento que atenda a saúde da gestante com intuito de educar e precaver. Cabe a equipe de saúde que saiba encaminhar essa criança pra atenção especializada, para que dessa forma o profissional possa diagnosticar e tratar as alterações orais e consequentemente seja estabelecida a atuação da equipe multidisciplinar.

É crucial que seja traçado um plano com elaborações de projetos que atendam as medidas preventivas primárias e secundárias promovendo o amadurecimento oportuno e adequado do sistema estomatognático, dessa forma, contribuindo para o desenvolvimento completo e normal da criança. E por consequência, promover uma melhor qualidade de vida para as mesmas. 


\section{Referências}

Aguiar, S. M. H. C. A. de; Castro, A. M. de; \& Barbierl, C. M. (2003). Alterações dentárias em crianças nascidas prematuras: revisão de literatura. J Bras Odontopediatr Odontol Bebê,.6(31), 240-243.

Aine, L., Backström, M. C., Mäki, R., Kuusela, A. L., Koivisto, A. M., Ikonen, R. S., et al. (2000) Enamel defects in primary and permanent teeth of children born prematurely. J Oral Pathol Med; 29:403-9.

Barbosa, D. M., Lemos, L. V., Banzi, E. C., \& Myaki, S. I. (2008) Prevalência de hipoplasia do esmalte em dentes decíduos de crianças nascidas prematuras. Rev Odontol Unesp; 37:261-5

Bertoncelli, N., Cuomo, G., Cattani, S., Mazz, C., Pugliese, M., Coccolini, E., et al. (2012) Oral Feeding Competences of Healthy Preterm Infants: A Review. International Journal of Pediatrics.

Brasil - Ministério da Saúde. DATASUS [homepage on the Internet]. Informações de Saúde - Estatísticas Vitais - Mortalidade e Nascidos Vivos: nascidos vivos desde 1994 http://tabnet. datasus.gov.br/cgi/deftohtm.exe?sinasc/cnv/nvuf.def

Brogårdh-Roth S, Stjernqvist K, \& Matsson L. (2008) Dental behavioural management problems and dental caries prevalence in 3- to 6-year-old Swedish children born preterm. Int J Paediatr Dent; 18:341-7.

Caixeta, F. F., \& Corrêa, M. S. (2005). Os defeitos do esmalte e a erupção dentária em crianças prematuras. Rev Assoc Med Bras ;51:195-9

Castro, C. R. S. (2014) Baixo peso ao nascer e condições de saúde bucal de crianças na primeira infância. Salvador; . [Tese de doutorado - Instituto de Saúde Coletiva da Universidade Federal da Bahia]

Castro, C. R. S, Cabral M. B. B. S., Mota E. L. A., Cangussu, M. C. T., \& Vianna M. I. P. (2019) Baixo peso ao nascer e atraso na erupção de dentes decíduos em crianças. Rev Bras Saúde Mater Infant. (3):711-21.

Chandra R. K. (2002) Nutrition and the immune system from birth to old age. Eur J Clin Nutr ;56:S73-6.

Cortines A. A. O., \& Costa L. R. (2016) Associated factors and persistence of palatal groove in preterm infants: a cohort study. BMC Pediatrics, 16:143.

Carvalho G. D. (2003) SOS respirador bucal: uma visão funcional e clínica da amamentação. Lovise.

Cruvinel V. R. N., Gravina, D. B. L., Azevedo, T. D. P. L., Bezerra, A. C. B., \& Toledo, A. O. (2010) Prevalence of dental caries and cariesrelated risk factors in premature and term children. Braz Oral Res., 4(3):329-35.

Dadalto, E. C. V., Marcon, C. W., Gomes, P. M., Sarmento, L. C., \& Rosa, E. M. (2018) Erupção do primeiro dente decíduo em lactentes nascidos pré-termo: acompanhamento de 12 meses. Rev odontol UNESP, 47 (3): 168 - 74.

Diniz, M. B., Coldebella, C. R., Zuanon, A. C. C., \& Cordeiro, R. C. L. (2011) Alterações orais em crianças prematuras e de baixo peso ao nascer: a importância da relação entre pediatras e odontopediatras. Revista Paulista de Pediatria., 29(3):449-55

Duke, P. M., Coulson, J. D., Santos, J. I., \& Johnson, J. D. (1976) Cleft palate associated with prolonged orotracheal intubation in infancy. J Pediatr, 89:9901 .

Eid R M R. (2001) Questões do dia-a.dia. Prematuridade e defeitos do esmalte. Revista APCD, 5 (3) :32.

Erenberg, A., \& Nowak, A. J. (1984) Palatal groove formation in neonates and infants with orotracheal tubes. Am J Dis Child, 138:974-5.

Fadavi, S., Punwani, I., \& Vidyasagar, D. (1993) Prevalence of dental caries in prematurelyborn children. J Clin Pediatr Dent, 17:16

Fadavi, S., Punwani, I. C., Adeni, S., \& Vidyasagar, D. (1992) Eruption pattern in the primary dentition of premature low-birth-weight children. ASDC J Dent, 59(2):120-2.

Ferrini, F. R. D. O., Marba, S. T. M., \& Gavião, M. B. D. (2007) Alterações bucais em crianças prematuras e com baixo peso ao nascer. Rev Paul Pediatr, 25 (1):66-71.

Figueiredo, C. M., Sampaio, J. N., \& Fischer, R. G. (2006) A doença periodontal como mecanismo de indução ao parto prematuro de crianças com baixo peso. Medcenter. com Odontologia. http://www.odontologia.com.br/artigos. asp?id=289\&idesp=15\&ler=s.

Grahnen H., Sjölin S., Stenström A. (1974) Mineralization defects of primary teeth in children born pre-term. Scand J Dent Res, 82 (5): 396-400.

Harila-Kaera V., Grön M., Heikkinen T., \& Alvesalo L. (2002) Sagittal occlusal relationships and asymmetry in prematurely born children. Eur J Orthod, 24: $615-25$.

Hegde R., \& Awan K. (2019) Effects of periodontal disease on systemic health. Dis Month, 65 (6): 185 - 192.

Impellizzeri A, Giannantoni I, Polimeni A, Barbato E, \& Galluccio G. (2019) Epidemiological characteristic of Orofacialclefts and its associated congenitalanomalies: retrospective study. BMC Oral Health, 19:290.

Koch G, Hallonstein A. L, Ludvigsson N, Hansson B, Holst A, \& Ullbro C. (1987) Epidemiologic study of idiopathic enamel hypomineralization in permanent teeth of Swedish children. Commun Dent Oral Epidemiol, 15 (2): 279-85.

Lafaurie, G. I., Gómez, L. A., Montenegro, D. A., Avila, J., Tamayo, M. C., Lancheros, M. C., Quiceno, J., Trujillo, T. G., Noriega, L. A., Grueso, M. L., \& Cepeda, K. (2020) Periodontal condition is associated with adverse perinatal outcomes and premature rupture of membranes in low-income pregnant women in Bogota, Colombia: a case-control study. J Matern Fetal Neonatal Med, 33 (1): 16 - 23. 
Machado, F. C., \& Ribeiro, R. A. (2004) Enamel defects and dental caries in premature and/ or low birthweight children. Pesq Bras Odontoped Clin Integr, 4: 243-7.

Neto, P. G. F., \& Falcão, M. C. (2014) Eruption chronology of the first deciduous teeth in children born prematurely with birth weight less than 1500 g. Rev Paul Pediatr, 32 (1):17-23.

Nicolau B, Marcenes W, Bartley M, \& Sheiham A. (2003) A life course approach to assessing causes of dental caries experience: the relationship between biological, behavioural, socio-economic and psychological conditions and caries in adolescents. Caries Res, 37: 319-26.

Paulsson L, Bondemark L, \& Soderfeldt B. (2004) A systematic review of the consequences of premature birth on palatal morphology, dental occlusion, toothcrown dimensions, and tooth maturity and eruption. Angle Orthod, 74: 269-79.

Pierce, K. M., Rozier, R. G., \& Vann Jr, W. F. (2002) Accuracy of pediatric primary care providers' screening and referral for early childhood caries. Pediatrics, 109:e82

Psoter, W. J., Rei, B. C., \& Katz, R. V. (2005) Malnutrition and dental caries: a review of the literature. Caries Res, 39 (6): $441-7$.

Rajshekar, A. S., \& Laxminarayan, N. (2011) Comparison of primary dentition caries experience in pre-term low birth-weight and full-term normal birthweight children aged one to six years. J Indian, 29 (2): 128-34.

Rother, E. T. (2007). Revisão sistemática x revisão narrativa. Acta Paulista de Enfermagem, 20 (2).

Saraiva, M. C., Bettiol, H., Barbieri, M. A., \& Silva, A. A. (2007) Are intrauterine growth restriction and preterm birth associated with dental caries? Community Dent Oral Epidemiol, 35: 364-76.

Saraiva, M. C., Chiga, S., Bettiol, H., Silva, A. A., \& Barbieri, M. A. (2007) Is low birthweight associated with dental caries in permanent dentition? Paediatr Perinat Epedemiol, 21:49-56.

Seow, W. K., Brown, J., Tudehope, D., \& Callaghan, M. (1984) Development defects in the primary dentition of lowbirth weight: adverse effects of laryngoscopy and prolonged endotracheal intubation. Pediatr Dent, 6 (1): 28-31.

Seow, W. K., Humphrys, C., Mahanonda, R., \& Tudehope, D. I. (1998) Dental eruption in low birth-weight prematurely born children: a controlled study. Pediatr Dent, 10: 39 - 42

Seow, W. K., \& Wan, A. A. (2000) controlled study of the morphometric changes in the primary dentition of preterm, very low-birthweight, children. $J$ Dent Res, 79: 63-9.

Seow W. K. (1991) Enamel hypoplasia in the primary dentition: a review. J Dent Child, 58 (6): 441 - 52.

Sheiham A. (1992) The role of the dental team in promoting dental health and general health through oral health. Int Dent J; $42: 223$ - 8.

Shibukawa, B. M. C., Rissi, G. P., Higarashi, I. H., \& Oliveira, R. R. (2021) Fatores associados à presença de fissura labial e/ou fenda palatina em recémnascidos brasileiros. 13 Rev Fac Odontol Univ Fed Bahia, 51(2).

Silveira, M. F., Santos, I. S., Matijasevich, A., Malta, D. C., \& Duarte, E. C. (2009) Nascimentos pré-termo no Brasil entre 1994 e 2005 conforme o Sistema de Informações sobre Nascidos Vivos (SINASC). Cad Saude Publica, 25: 1267- 75.

Van Der Laan T. (1995) A importância da amamentação no desenvolvimento facial infantil. Pró-fono, 7:3 - 5.

Viana, K. A., Euzebio, L. F., Cortines, A. A. O., \& Costa, L. R. (2013) Hábitos relacionados à saúde bucal em crianças nascidas prematuras: uma preocupação para equipes multiprofissionais de saúde. Arq Odontol. 49 (3): 140 - 6.

Viscardi, R. M., Romberg, E., \& Abrams, R. G. (1994) Delayed primary tooth eruption in premature infants: relationship to neonatal factors. Pediatr Dent, 16: $23-8$.

Wan, A. K., Seow, W. K., Purdle, D. M., Bird, P. S., Walsh, L. J., \& Tudehope, D. I. (2003) Immunoglobulins in saliva of preterm and full-term infants. A longitudinal study from 0-18 months of age. Oral Microbiol Immunol 18: 72 - 8.

Williams, C. E., Davenport, E. S., Sterne, J. A., Sivapathasundaram, V., Fearne, J. M., \& Curtis, M. A. (2000) Mechanisms of risk in preterm low-birthweight infants. Periodontol, 23: $142-50$. 